\title{
Radiological assessment of periapical healing using the cone beam computed tomography periapical index: case report
}

\author{
Dr. Ambica Khetarpal ${ }^{1}$, Dr. Sarika Chaudhary ${ }^{2}$, Dr Sharad Sahai ${ }^{3}$, \\ Dr. Sangeeta Talwar ${ }^{4}$, Dr Mahesh Verma ${ }^{5}$ \\ ${ }^{I}$ (Department of Conservative Dentistry and Endodontics, Maulana Azad Institute of Dental Sciences, Bahadur \\ Shah Zafar Marg, New Delhi - 110002, New Delhi, India) \\ ${ }^{2}$ (Department of Conservative Dentistry and Endodontics, Maulana Azad Institute of Dental Sciences, Bahadur \\ Shah Zafar Marg, New Delhi-110002, New Delhi, India) \\ ${ }_{3}^{3}$ (Department of Oral amd maxillofacial Rdaiology, Diwan Chand Diagnostic and Radiology Centre, New Delhi \\ - 110002, New Delhi, India) \\ ${ }^{4}$ (Department of Conservative Dentistry and Endodontics, Maulana Azad Institute of Dental Sciences, Bahadur \\ Shah Zafar Marg, New Delhi - 110002, New Delhi, India) \\ ${ }^{5}$ (Department of Conservative Dentistry and Endodontics, Maulana Azad Institute of Dental Sciences, Bahadur \\ Shah Zafar Marg, New Delhi - 110002, New Delhi, India)
}

\begin{abstract}
Periapical radiography is essential in diagnosis, treatment planning and follow-up in endodontics. However, image interpretation can be confounded by geometric distortion, superimposition of regional anatomy and technique errors. These problems can be overcome by utilizing small or limited-volume cone beamcomputed tomography imaging techniques, which produce accurate 3-D images of the teeth and surrounding dentoalveolar structures. The periapical index score (PAI) is commonly used to follow up the lesions in the bone using periapical radiographs. Recently, a new PAI scoring system based on CBCT was introduced (CBCT-PAI). We report a case where CBCT-PAI scoring was achieved using image fusion to assess healing of a large periapical lesion.
\end{abstract}

Keywords: Apical periodontitis, cone beam computed tomography, healing, periapical index, radiography

\section{Introduction}

Bone repair or periapical lesion progression after endodontic therapy of teeth with chronic periapical lesions is usually monitored in a subjective manner using conventional radiographs, which does not assure the precise identification of periapical lesion changes or their extension[1]. The diagnosis of apical periodontitis (AP) represents an essential strategy to determine the treatment plan. Although apical periodontitis can be accurately identified by histological analysis [2]; CBCT can be a noninvasive diagnostic choice[3, 4]. It has been demonstrated that apical periodontitis can be detected sooner and more predictably by using CBCT than by using periapical radiographs [5-8]. CBCT can be used for more precise radiographic evaluation of periapical lesion areas measured in different follow-up periods, owing to the method's ability to detect extremely small bone changes. CBCT has been successfully used in endodontics with different goals, including study of root canal anatomy, external and internal macromorphology in 3-dimensional reconstruction of the teeth, evaluation of root canal preparation, obturation, re-treatment, coronal microleakage, detection of bone lesions, and experimental endodontology[7, 9-11]. CBCT provides promising results with a more accurate detection of AP [7, 9-11]. It can provide more accuracy in the assessment of bone formation or resorption during or after root canal treatment.

Radiographic examination is a common procedure used to determine the outcome of root canal therapy. Previous studies $[12,13]$ have referred to the periapical index (PAI) as a scoring system for radiographic assessment of AP. The commonly used PAI system relies on the comparison of periapical radiographs with a set of five radiographic images derived from Brynolf's histological-radiographic correlation study $[12,14]$. However, the validity of using the PAI for all teeth might be questionable as the thickness of the cortical bone and the position of the root tips in relation with the cortex varies with the position of the tooth. Also, the conventional radiographs used for the management of endodontic problems yield limited information because of the two-dimensional (2D) nature of the images produced, geometric distortion and anatomical noise. In 2008, Estrela et $\mathrm{Al}$ [8] introduced a new PAI based on CBCT (CBCT-PAI). It offers an accurate diagnostic method for use with high-resolution images, which can reduce the incidence of false negative diagnosis, minimize observer interference, and increase the reliability of epidemiologic studies, especially those referring to AP prevalence and severity. 
The current case report evaluates the healing of a large periapical lesion after nonsurgical endodontic therapy using image fusion and $\mathrm{CBCT}$ periapical index (CBCT-PAI).

\section{Case Report}

A 23-year old man was referred complaining of repeated swelling and pain in the anterior maxilla over the last few months. Clinical examination revealed that left maxillary incisors were tender to percussion and palpation and the central incisor had open access cavity. A localized, erythematous swelling was present on the buccal and palatal mucosa surrounding the teeth. The periodontal status of all maxillary anterior teeth was normal (probing depth $<3 \mathrm{~mm}$ ), and none were mobile. Radiographs revealed that maxillary left incisors had a large radiolucency in the periapical region (Fig 1). A diagnosis of pulpal necrosis with symptomatic apical abscess because of inadequate previous treatment was made after corroboration of clinical and radiological findings. The medical history of the patient was noncontributory. It was hence decided to endodontically retreat the incisors. CBCT was also planned for three dimensional assessment of the lesion.

Following rubber dam isolation and local anaesthesia using 2\% lidocaine and 1:100,000 epinephrine, the outline of the access cavity in the central incisor was modified with a diamond coated size 10 fissure bur (Diatech, Heerbrugg, Switzerland) in a high-speed handpiece. Endodontic access cavity preparation was also performed in the lateral incisor by using Endo-Access diamond bur (Dentsply, Maillefer, Baillaigues, Switzerland) under local anesthesia, and a periapical radiograph was taken to determine the working length. The root canals were prepared using ProTaper rotary files (Dentsply Maillefer, Ballaigues, Switzerland) till F5 finishing file and irrigated passively with $20 \mathrm{ml}$ of $5.25 \% \mathrm{NaOCl}$. As there was continuous exudate from the root canal, radiopaque calcium hydroxide powder was mixed with saline to a creamy consistency and placed inside the canal space using a gentle counterclockwise motion of a size $50-\mathrm{K}$ file to the radiographically estimated working length (Fig 2); the teeth were then temporarily restored with Coltosol (Cavit G; 3M ESPE, Seefeld, Germany). Obturation was done for both the teeth in the subsequent visit (Fig 3).

CBCT images for the maxillary anterior region were obtained before and after endodontic intervention (CS9300, Carestream Healthcare India Pvt Ltd) in dental mode at $84 \mathrm{kV}, 5 \mathrm{~mA}$. A single scout image, i.e. lateral view was taken in accordance with the patient's position, and a $360^{\circ}$ scan was acquired afterwards. The total scan time was $20 \mathrm{~s}$. Images were reconstructed from the volumetric dataset, in planes perpendicular to the selected tooth axes. True and oblique axial, coronal and sagittal images with a thickness of $0.09 \mathrm{~mm}$ and an interval of $0.09 \mathrm{~mm}$ were obtained. Cross-sectional images with a thickness of $0.09 \mathrm{~mm}$ and an interval of $1 \mathrm{~mm}$ were also prepared. Image assessment was performed by a calibrated endodontist and a maxillofacial radiologist using the CBCT software tools. Assessment included evaluation of the extent or size, margins, internal structure of the lesion and effects on surrounding teeth or anatomic structures in multiple reformatted image planes by using the working tools of Xelis Dental (Infinitt Inc. Seoul, Korea) and Ondemand 3D (Cybermed Inc. Seoul, Korea) (Fig 2).

CBCT scan was repeated after 10 months to assess the healing of periapical lesion using the CBCTPAI. All scan parameters were identical to the pre-treatment CBCT. The CBCT-PAI was determined by assessment of the greatest antero-posterior, transverse and supero-inferior dimensions of the lesion. A 6-point (0 - 5) scoring system was used. In addition, considering that CBCT provides 3-dimensional images, with depth being added as a new plane of analysis, 2 variables were included in the scoring system as appropriate, expansion of cortical bone (E) and destruction of cortical bone (D) (TABLE 1 and Figs. 4-6). The pre-treatment CBCT examination revealed a CBCT-PAI score $5+\mathrm{E}+\mathrm{D}$ as the major diameter of the lesion was approximately $15 \mathrm{~mm}$ along with thinning, expansion and partial erosion of the adjoining labial cortical plate in tooth \#11 region. The post-treatment CBCT-PAI score was determined to be Score 4.

We used third party image processing software (Ondemand 3D, Cybermed Inc., Seoul, Korea) for reproducible superimposition or fusion of the pre-and post-operative CBCT volumetric datasets (Fig 7, 8).

\section{Discussion}

Clinical and radiologic criteria are frequently used to assess the status of endodontic treatment and its correlation with AP $[1-4,9,10,12,13]$, but morphologic variations, surrounding bone density, x-ray angulations, and radiographic contrast can influence radiographic interpretation $[10,12]$.

Two-dimensional radiographs can image periapical lesions to be detected only when bone mineral loss reaches $30 \%-50 \%$ due to structured noise of superimposed cortices [15]. Therefore, only $50-55 \%$ of small and medium sized lesions can be diagnosed as periapical disease [16]. Reports indicate that CBCT images provide clinically relevant information not found in periapical images [17-20].

The Periapical Index as indicated by Ørstavik et al. [12] ranges from health to severe periodontitis on the basis of the interpretation of 2-dimensional radiographic images, whereas CBCTPAI scores have been established according to the interpretation of 3-dimensional CBCT scans. 
The Cone Beam Computed Tomography Periapical Index (CBCTPAI) used in this study was based on the criteria established from measurements corresponding to periapical radiolucency interpreted on CBCT scans. CBCTPAI scores were calculated by analysis of the lesion in 3 dimensions. The measurement of lesion depth contributed significantly to diagnosis and improved prognosis. Limitations of methodology used in previous studies include arbitrary orientation of the three orthogonal planes for measurements and lack of fixed parameters to select the larger diameter of the lesion [21]. These were eliminated by auto-registration of the preoperative and post-operative scan datasets in axial, sagittal and coronal planes using the 'Fusion' module in Ondemand 3D software. The tool also permitted visualization of change in size of the lesion due to endosteal new bone formation, labial recortication. The results of the present study also show that $\mathrm{CBCT}$ was a reliable method to detect the healing of periapical lesions after root canal treatment, showing periapical bone changes over small time intervals.

The CBCT-PAI can reduce the incidence of false negative diagnosis, minimize observer interference, and increase the reliability of epidemiologic studies, especially those referring to AP prevalence and severity.

In this case, the periapical healing was better appreciated using CBCT and image fusion with a significant reduction in the CBCT-PAI from Score 5+E+D to Score 4. Therefore, fusion of pre- and posttreatment $\mathrm{CBCT}$ scans can provide an accurate and reproducible method for estimation of CBCT - PAI.

\section{Figures}

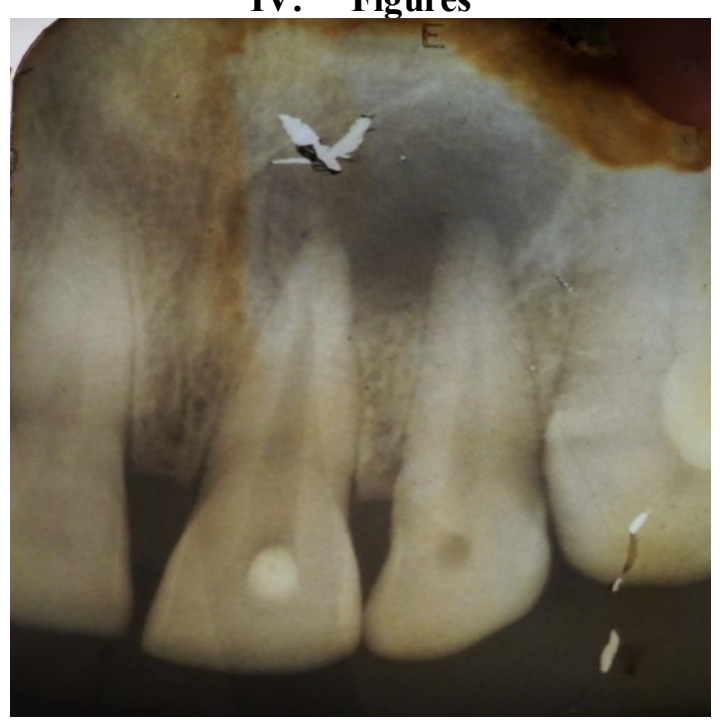

figure 1- preoperative radiograph

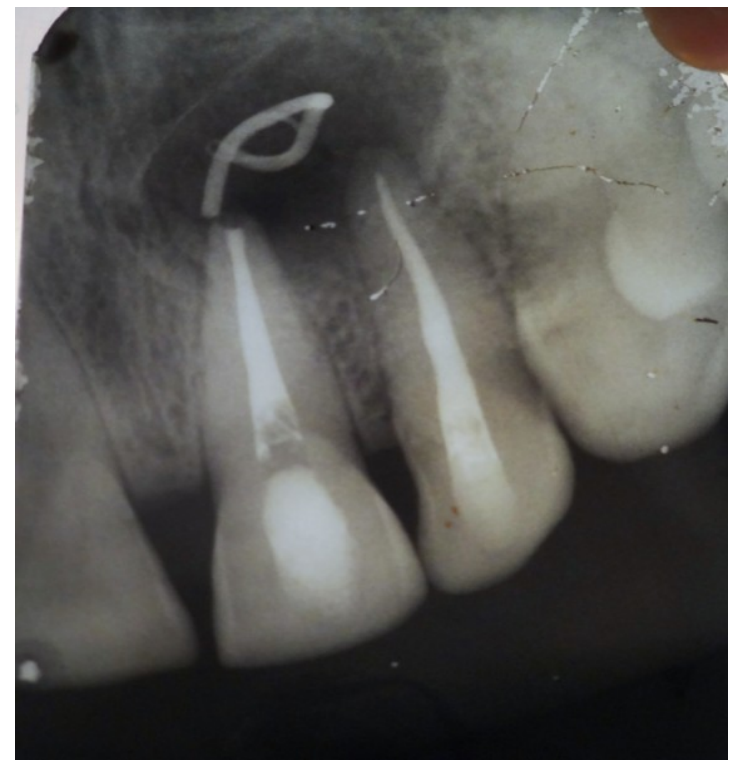

figure 2- post-obturation radiograph 


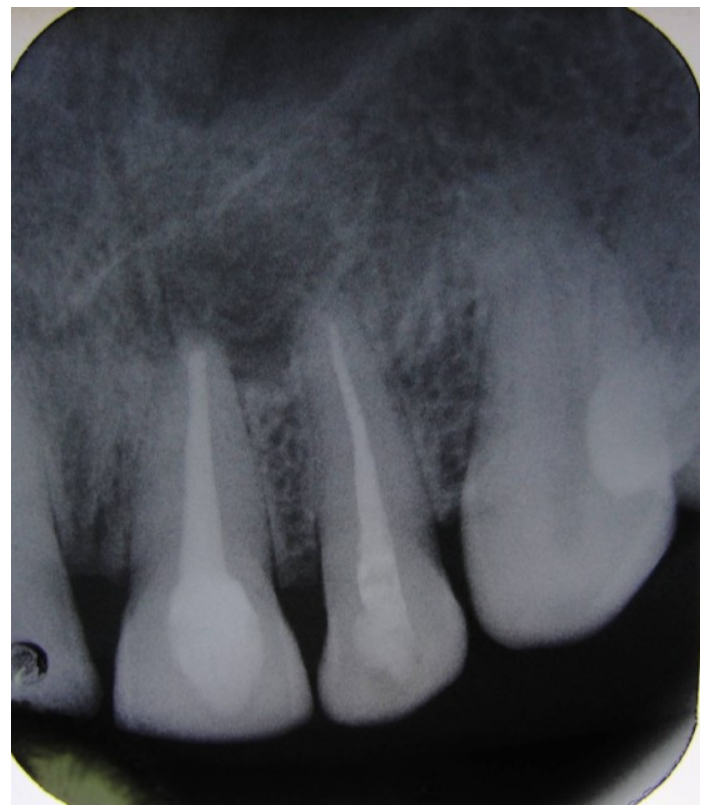

figure 3- periapical radiograph after 10 months

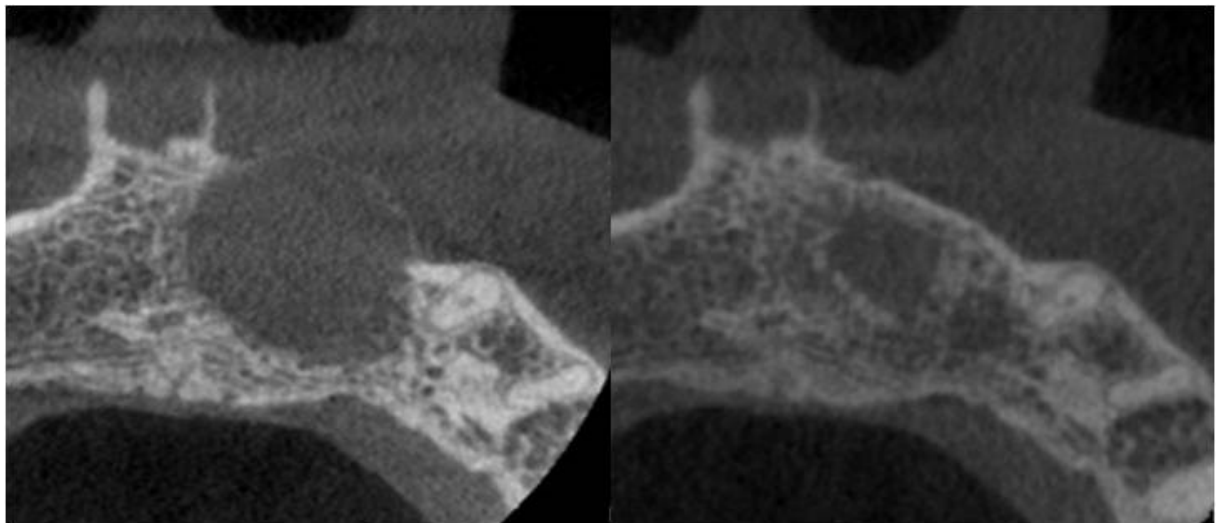

figure 4 - pre-operative (left) and post-operative (right) axial image sections obtained at the level of periapical region of teeth \#11, 12. Pre-operative image shows an ovoid osteolytic lesion without internal calcifications or septae, thinning and expansion of adjoining labial cortical plate. post-operative image shows reduced size of lesion due to new bone formation, re-cortication of adjoining labial plate.

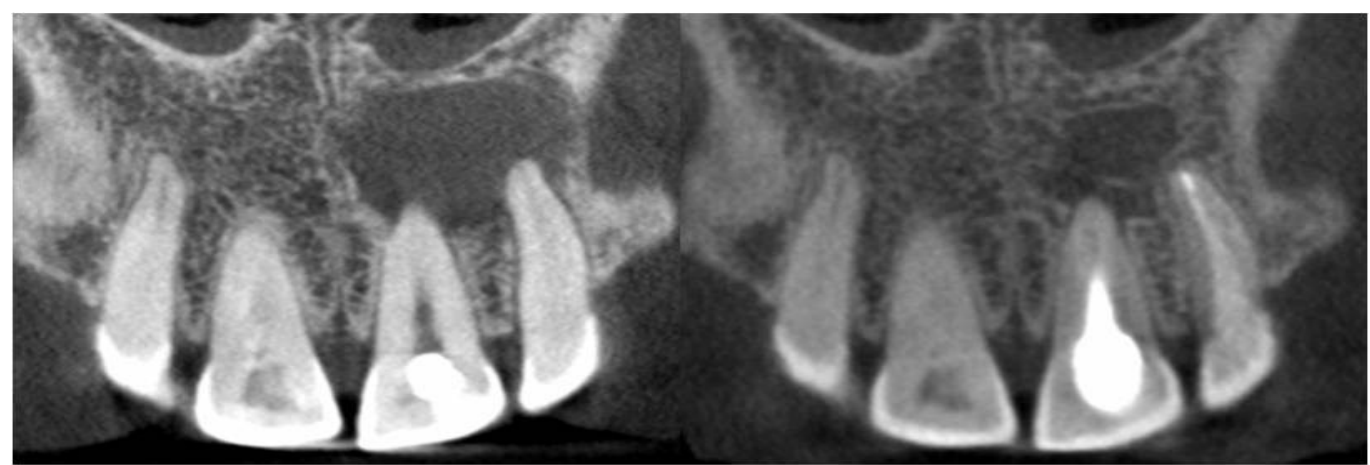

figure 5 - pre-operative (left) and post-operative (right) oblique coronal image sections obtained through the anterior maxilla. Pre-operative image shows an ovoid osteolytic lesion with distinct margins extending till intermaxillary suture medially and till the level of nasal fossa floor cranially. post-operative image shows reduced size of lesion and endodontic restorations in-situ with \#11, 12. 


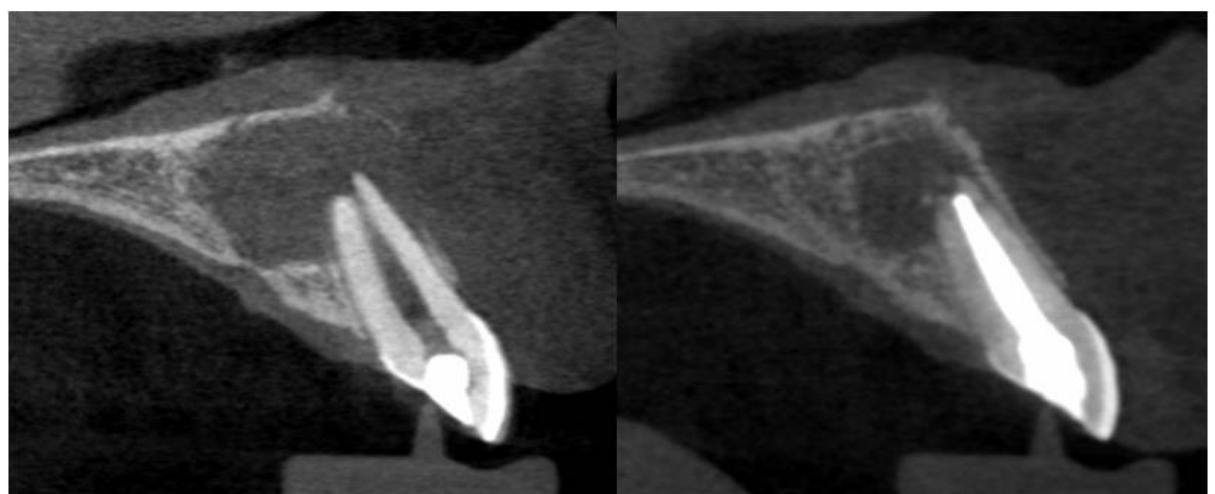

figure 6 - pre-operative (left) and post-operative (right) sagittal image sections obtained through the \#11 region of maxilla. Pre-operative image shows a large osteolytic lesion extending from the middle third of alveolus till nasal fossa floor with loss of labial cortical plate and focal thinning of the palatal cortex. apical blunting and root resorption is also seen with \#11. Post-operative image shows labial re-cortication, reduced size of lesion due to endosteal new bone formation and endodontic restorations in-situ with \#11.

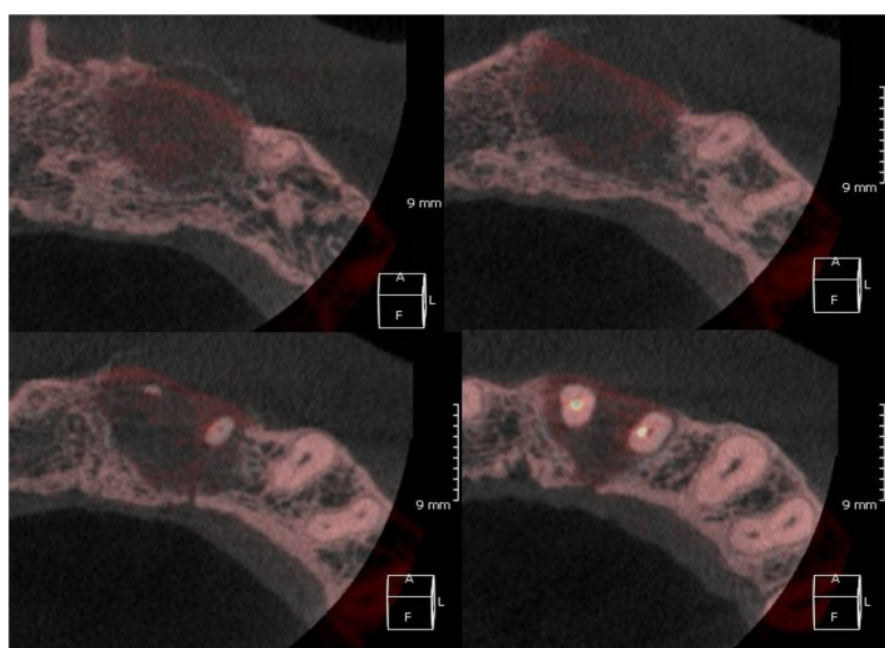

figure 7 - fused pre-operative and post-operative axial image sections from periapical region till apical third of roots of \#11, 12 (clockwise top left to bottom right). Labial recortication and endosteal new bone formation can be seen against background of pre-treatment lesion.

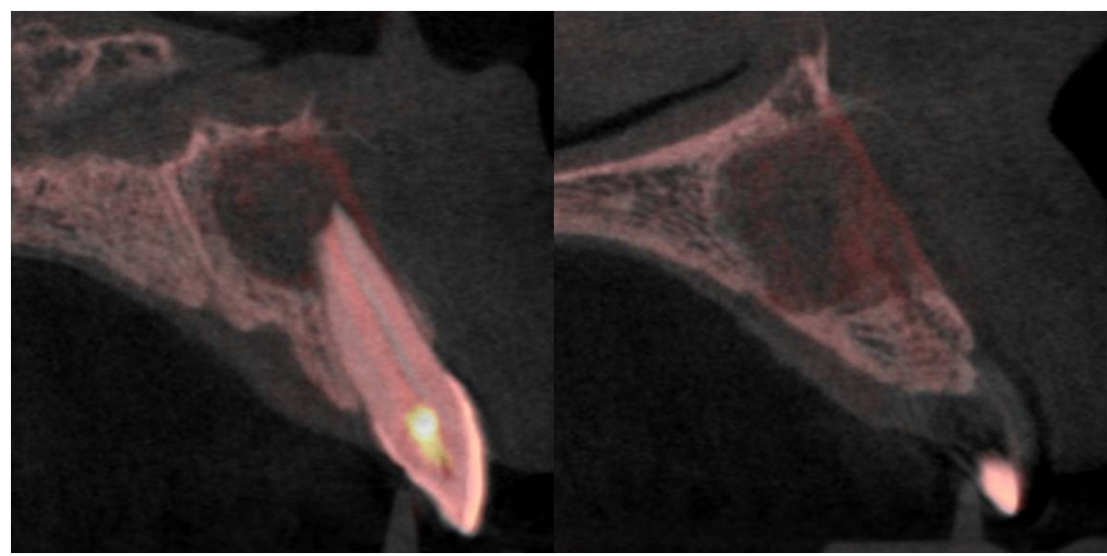

figure 8 - fused pre-operative and post-operative sagittal image sections from \#11, 12 region (1 to r). labial recortication and endosteal new bone formation can be seen against background of large pre-treatment lesion size and expanded, partially deficient labial cortical plate. 


\section{Table 1. Cone Beam Computed Tomography Periapical Index Scores Score Quantitative Bone Alterations in Mineral Structures}

\begin{tabular}{|c|c|}
\hline & Intact periapical bone structures \\
\hline 1 & Diameter of periapical radiolucency " $0.5-1 \mathrm{~mm}$ \\
\hline & Diameter of periapical radiolucency " $1-2 \mathrm{~mm}$ \\
\hline & Diameter of periapical radiolucency " $2-4 \mathrm{~mm}$ \\
\hline & Diameter of periapical radiolucency " $4-8 \mathrm{~mm}$ \\
\hline & Diameter of periapical radiolucency " $8 \mathrm{~mm}$ \\
\hline
\end{tabular}

\# $\mathrm{E}^{*}$

Expansion of periapical cortical bone

Score (n)

\# D*

Destruction of periapical cortical bone

*The variables E (expansion of cortical bone) and D (destruction of cortical bone) were added to each score, if either of these conditions was detected in the CBCT analysis.

\section{Conclusion}

Image fusion and CBCT periapical index (CBCT-PAI) can prove as an important tool for assessment of healing of a large periapical lesion after nonsurgical endodontic therapy. It is an accurate diagnostic method which can limit the incidence of false negative diagnoses.

\section{References}

[1]. Cotti E, Vargiu P, Dettor C, Mallarini G. Computerized tomography in the management and follow-up of extensive periapical lesion. Endod Dent Traumatol, 15, 186-189.

[2]. Laux M, Abbott PV, Pajarola G, Nair PNR. Apical inflammatory root resorption: a correlative radiographic and histological assessment. Int Endod J, 33, 2000, 483-93.

[3]. Trope M, Pettigrew J, Petras J, Barnett F, Tronstad L. Differentiation of radicular cyst and granulomas using computerized tomography. Endod Dent Traumatol, 5, 1989, 69-72.

[4]. Simon JHS, Enciso R, Malfaz JM, Rogers R, Bailey-Perry M, Patel A. Differential diagnosis of large periapical lesions using cone beam computed tomography measurements and biopsy. J Endod, 32, 2006,833-7.

[5]. Cotti E. Advanced techniques for detecting lesions in bone. Dent Clin North Am, 54, 2010, 215-235.

[6]. Patel S. New dimensions in endodontic imaging: Part 2. Cone beam computed tomography. Int Endod J, 42, 2009, 463-475.

[7]. Lofthag-Hansen S, Hummonen S, Grondahl K. Limited cone beam CT and intraoral radiography for the diagnosis of periapical pathology. Oral Surg Oral Med Oral Pathol Oral Radiol Endod, 103, 2007, 114-119.

[8]. Estrela C, Bueno MR, Azevedo BC, Azevedo JR, Pe'cora JD. A new periapical index based on cone beam computed tomography. JEndod 34, 2008, 1325-1331.

[9]. Velvart P, Hecker H, Tillinger G. Detection of the apical lesion and the mandibular canal in conventional radiography and computed tomography. Oral Surg Oral Med Oral Pathol Oral Radiol Endod, 92, 2001, 682- 8.

[10]. Nakata K, Naitoh M, Izumi M, Inamoto K, Ariji E, Nakamura H. Effectiveness of dental computed tomography in diagnostic imaging of periradicular lesion of each root of a multirooted tooth: a case report. $J$ Endod, 32, 2006, 583-7.

[11]. Cotton TP, Geisler TM, Holden DT, Schwartz SA, Schindler WG. Endodontic applications of cone-beam volumetric tomography. JEndod, 33, 2007, 1121-32.

[12]. Ørstavik D, Kerekes K, Eriksen HM. The periapical index: a scoring system for radiographic assessment of apical periodontitis. Endod Dent Traumatol, 2, 1986, 20-4.

[13]. Reit C, Grøndahl HG. Application of statistical decision theory to radiographic diagnosis of endodontically treated teeth. Scand $J$ Dent Res, 9, 1983, 213-8.

[14]. Brynolf I. A histologic and roentgenologic study of the periapical region of human upper incisors. Odontol Revy, 18, 1967, 1-176

[15]. Bender IB. Factors influencing the radiographic appearance of bony lesions. J Endod, 23, $1997,5-14$.

[16]. Cavalcanti MGP, Ruprecht A, Johnson WT, Southard TE, Jakobsen J. The contribution of trabecular bone to the visibility of the lamina dura: an in vitro radiographic study. Oral Surg Oral Med Oral Pathol Oral Radiol Endod, 93, 2002, $118-122$.

[17]. Low KMT, Dula K, Burgin WB, von Arx T. Comparison of periapical radiography and limited cone-beam tomography in posterior maxillary teeth referred for apical surgery. J Endod, 34, 2008, 557-62.

[18]. Sogur E, Grondahl HG, Baksi BG, Mert A. Does a combination of two radiographs increase accuracy in detecting acid-induced periapical lesions and does it approach the accuracy of cone-beam computed tomography scanning? J Endod, 38, 2012,131-136.

[19]. Ordinola-Zapata R, Bramante CM, Duarte MH, Ramos Fernandes LM, Camargo EJ, de Moraes IG, Bernardineli N, et al. The influence of cone-beam computed tomography and periapical radiographic evaluation on the assessment of periapical bone destruction in dog's teeth. Oral Surg Oral Med Oral Pathol Oral Radiol Endod, 112, 2011, 272-79.

[20]. Bornstein MM, Lauber R, Sendi P, von Arx T. Comparison of periapical radiography and limited cone-beam computed tomography in mandibular molars for analysis of anatomical landmarks before apical surgery. J Endod, 37, 2011, 151-57.

[21]. Esposito S, Cardaropoli M, Cotti E. A suggested technique for the application of the cone beam computed tomography periapical index. Dentomaxillofac Radiol. 40, 2011, 506-12. 